\title{
Minimal Logarithmic Signatures for one type of Classical Groups
}

\author{
MLSs for one type of Classical Groups
}

\author{
Haibo Hong • Licheng Wang • Haseeb \\ Ahmad · Yixian Yang
}

Received: date / Accepted: date

\begin{abstract}
As a special type of factorization of finite groups, logarithmic signature (LS) is used as the main component of cryptographic keys for secret key cryptosystems such as PGM and public key cryptosystems like $M S T_{1}$, $M S T_{2}$ and $M S T_{3}$. An LS with the shortest length, called a minimal logarithmic signature (MLS), is even desirable for cryptographic applications. The MLS conjecture states that every finite simple group has an MLS. Recently, the conjecture has been shown to be true for general linear groups $G L_{n}(q)$, special linear groups $S L_{n}(q)$, and symplectic groups $S p_{n}(q)$ with $q$ a power of primes and for orthogonal groups $O_{n}(q)$ with $q$ as a power of 2 . In this paper, we present new constructions of minimal logarithmic signatures for the orthogonal group $O_{n}(q)$ and $S O_{n}(q)$ with $q$ as a power of odd primes. Furthermore, we give constructions of MLSs for a type of classical groups - projective commutator subgroup $P \Omega_{n}(q)$.
\end{abstract}

Keywords (Minimal) logarithmic signature - Orthogonal group · Projective commutator subgroup · Stabilizer $\cdot$ Spreads

Mathematics Subject Classification (2000) MSC 94A60 - MSC 94A60 . MSC 11T71 · MSC 14G50 · MSC 20G40 • MSC 20E28 · MSC 20E32 · MSC . MSC 20D06 · MSC 05E15 · MSC 51A40

\section{Introduction}

The security of many public key cryptosystems is based on the hardness assumptions of certain problems over large finite abelian algebraic structures such as cyclic groups, rings and finite fields. Two well-known hard problems are integers factorization problem (IFP) and discrete logarithm problem (DLP).

Information Security Center, State Key Laboratory of Networking and Switching Technology, Beijing University of Posts and Telecommunications, Beijing, 100876 P.R. China E-mail: honghhaibo1985@163.com wanglc@bupt.edu.cn 
However, these hardness assumptions would be broken if quantum computers become practical. For instance, Shor's quantum algorithms [17] solve IFP and DLP very efficiently. The security status of currently used cryptosystems, mainly based on IFP and DLP or their variants, becomes even worse due to the known great progress on finding possible solutions for building quantum computers on practical scales.

Therefore, it is imminent to design effective and practical cryptographic schemes that have the potential for resisting quantum algorithm attacks. Actually, several attempts using non-abelian algebraic structures were made and some available cryptographic schemes such as $P G M, M S T_{1}, M S T_{2}$ and $M S T_{3}$ 3, 16, 14, 11, 9, 24 were developed during the past decades. In particular, as a natural analogy of the hardness assumption of IFP, the group factorization problem (GFP) [10, 15] and its hardness assumption over certain factorization basis, referred as logarithmic signature, play a core role in the security arguments for the family of $M S T$ cryptosystems.

Security is not the unique goal of designing a cryptosytem. Instead, efficiency is also a major issue. With the purpose for minimizing the parameter sizes, a natural question comes to mind: How to make the factorization basis known as logarithmic signature (LS), as short as possible in the family of MST cryptosystems? A minimal logarithmic signature (MLS) is an LS with the shortest length. In other word, further shorten an MLS would make it no longer an LS. New question arises: Does any finite (non-abelian) group has MLSs?

In fact, some encouraging work has been done in searching the MLSs for finite groups. According to the pioneering work due to Vasco et al. [18, Holmes 20] and Lempken et al. 8, we know that, with few exceptions, MLSs exist for all groups of order $\leq 10^{10}$. Most recently, Nikhil Singhi, Nidhi Singhi, and Magliveras [21,23 made another breakthrough: MLSs exist for the groups $G L_{n}(q), S L_{n}(q), S p_{n}(q)$ with $q$ as a power of a prime and $O_{n}(q)$ with $q$ as a power of 2. As far as we know, this is the first result not constrained by a specified boundary on group orders. Besides, Nikhil Singhi and Nidhi Singhi 23 also pointed out, without any proof, that the MLSs should also exist for $O_{n}(q)$ with $q$ as a power of odd primes.

Therefore, in this paper, our main motivation is to present new constructions of minimal logarithmic signatures for the orthogonal group $O_{n}(q)$, the special orthogonal group $S O_{n}(q)$, the projective special orthogonal group $\operatorname{PSO}_{n}(q)$, the commutator subgroup $\Omega_{n}(q)$ and one type of classical groups [5] - projective commutator subgroup $P \Omega_{n}(q)$ with $q$ as a power of odd primes. For $O_{n}(q)$ and $S O_{n}(q)$, the proposed MLSs have the similar structure $\left[A, B^{\prime}, G_{w}\right]$, where $A=\langle a\rangle$, and $B^{\prime}=\{h C \mid h \in B\}, C \leq B, B=\langle b\rangle$, while $G_{w}=P: Q$ is a semi-direct product of a $p$-group $P$ and a direct product $Q=G L_{1}(q) \times Y$ (see Table 1). We employ two canonical homomorphisms $\eta: S O_{n}(q) \rightarrow P S O_{n}(q)$ and $\theta: \Omega_{n}(q) \rightarrow P \Omega_{n}(q)$ for proving the existence of MLSs for $P S O_{n}(q)$ and $P \Omega_{n}(q)$, respectively.

The rest of contents are organized as follows: Necessary preliminaries are presented in Section 2; In Section 3, we utilize the Levi decomposition of 
Table 1 MLSs for $O_{n}(q)$ and $S O_{n}(q)$

\begin{tabular}{|c|c|c|c|c|}
\hline & $A=\langle a\rangle$ & $\begin{array}{l}B^{\prime}=\{h C \mid h \in B\} \text { with } \\
C \leq B=\langle b\rangle,|C|=q-1\end{array}$ & \multicolumn{2}{|c|}{$\begin{array}{l}G_{w}=P: Q \text { with } \\
Q=G L_{1}(q) \times Y\end{array}$} \\
\hline & $a$ & $b$ & $|P|$ & $Y$ \\
\hline \multirow{3}{*}{$O_{n}(q)$} & $x_{1}^{q^{m}-1}$ for $x_{1} \in G L_{2 m}(q)$ & $\left(\begin{array}{cccc}D_{1} & 0 & 0 & 0 \\
0 & 1 & 0 & 0 \\
0 & 0 & \left(D_{1}^{t}\right)^{-1} & 0 \\
0 & 0 & 0 & 1\end{array}\right)$ for $D_{1} \in G L_{2 m-2}(q)$ & $q^{2 m-2}$ & $O_{2 m-2}^{-}(q)$ \\
\hline & $x_{2}^{q^{m-1}-1}$ for $x_{2} \in G L_{2 m}(q)$ & $\left(\begin{array}{cc}D_{2} & 0 \\
0 & \left(D_{2}^{t}\right)^{-1}\end{array}\right)$ for $D_{2} \in G L_{2 m}(q)$ & $q^{2 m-2}$ & $O_{2 m-2}^{+}(q)$ \\
\hline & $x_{3}^{q^{m}-1}$ for $x_{3} \in G L_{2 m+1}(q)$ & $\left(\begin{array}{ccc}D_{3} & 0 & 0 \\
0 & \left(D_{3}^{t}\right)^{-1} & 0 \\
0 & 0 & 1\end{array}\right)$ for $D_{3} \in G L_{2 m}(q$ & $q^{2 m-1}$ & $O_{2 m-1}(q)$ \\
\hline \multirow{3}{*}{$S O_{n}(q)$} & $x_{1}^{* q^{m}-1}$ for $x_{1}^{*} \in O_{2 m}^{-}(q)$ & $\left(\begin{array}{cccc}D_{1}^{*} & 0 & 0 & 0 \\
0 & 1 & 0 & 0 \\
0 & 0 & D_{1}^{* t} & 0 \\
0 & 0 & 0 & 1\end{array}\right)$ for $D_{1}^{*} \in O_{m-1}(q)$ & $q^{2 m-2}$ & $S O_{2 m-2}^{-}(q)$ \\
\hline & $x_{2}^{* q^{m-1}-1}$ for $x_{2}^{*} \in O_{2 m}^{+}(q)$ & $\left(\begin{array}{cc}D_{2}^{*} & 0 \\
0 & D_{2}^{* t}\end{array}\right)$ for $D_{2}^{*} \in O_{m}(q)$ & $q^{2 m-2}$ & $S O_{2 m-2}^{+}(q)$ \\
\hline & $x_{3}^{* q^{m}-1}$ for $x_{3}^{*} \in O_{2 m+1}(q)$ & $\left(\begin{array}{ccc}D_{3}^{*} & 0 & 0 \\
0 & D_{3}^{* t} & 0 \\
0 & 0 & 1\end{array}\right)$ for $D_{3}^{*} \in O_{m}(q)$ & $q^{2 m-1}$ & $S O_{2 m-1}(q)$ \\
\hline
\end{tabular}

parabolic subgroups to construct LSs for the parabolic subgroups of $O_{n}(q)$ and $S O_{n}(q)$; In Section 4, analogous to methods in 23, we use the totally isotropic subspaces to prove the existence of MLSs for $O_{2 m}^{-}(q)$ and $S O_{2 m}^{-}(q)$; In Section 5, we utilize suitable spread in the $P(V)$ [2,7,12] to accomplish the proof for $O_{2 m}^{+}(q)$ and $S O_{2 m}^{+}(q)$; In Section 6, we make further efforts to present the constructions of MLSs for $P S O_{2 m}^{ \pm}(q)$ and $P \Omega_{2 m}^{ \pm}(q)$; In Section 7, we take account of MLSs for $O_{2 m+1}(q), S O_{2 m+1}(q), P S O_{2 m+1}(q), \Omega_{2 m+1}(q)$ and $P \Omega_{2 m+1}(q)$.

\section{Preliminaries}

\subsection{Classical Spreads and Quadratic Spaces in Finite Fields}

Let $K$ be a finite field and $V$ a $n$-dimensional vector space over $K$. For $v_{1} \in V$, $\left\langle v_{1}\right\rangle$ denotes the one-dimensional subspace generated by $v_{1} . P(V)$ denotes the projective space on $V$, which is the set of all one-dimensional subspaces of $V$ [13.

Now, we describe the classical spread [12,7,4,2,23. An r-partial spread in $V$ is a set $S=\left\{W_{i} \mid 1 \leq i \leq t\right\}$ of $r$-dimensional subspaces $W_{i}$ such that $W_{i} \cap W_{j}=\langle 0\rangle$ for $i \neq j$. If $\cup_{i=1}^{t} W_{i}=V$, then $S$ is an $r$-spread in $V$. Besides, when $S$ is an $r$-(partial) spread in $P(V)$, it partitions $P(V)$ into $(r-1)$-dimensional subspaces of $P(V)$.

Suppose that $V=F_{q^{2 m}}$ is a finite field, $\alpha$ is a primitive element of field $F_{q^{2 m}}$ and $W=F_{q^{m}}$ is an $m$-subspace of $V$. For every $x \in V, W_{x}=\{w x \mid w \in W\}$, 
the set $S=\left\{W_{x} \mid x \in V\right\}$ forms a $m$-spread in $V$ 23. Meanwhile, we have the following remark.

Remark 1 21,23. Let $W_{i}=W \alpha^{\left(q^{m}-1\right) i}=\left\{w \alpha^{\left(q^{m}-1\right) i} \mid w \in W\right\}, 0 \leq i \leq q^{m}$. Then, the spread $S$ can also be described as $S=\left\{W_{i} \mid 0 \leq i \leq q^{m}\right\}$.

Let $V$ be an $n$-dimensional vector space over the finite field $K=F_{q}$ with $q=p^{e}$ for some prime $p$ and a positive integer $e$. Let $\mathcal{B}=\left\{e_{1}, \cdots, e_{n}\right\}$ be an ordered basis for $V$. Then a bilinear form over a vector space $V$ is a map $f: V \times V \rightarrow K$ satisfying:

$$
\begin{aligned}
& f(\lambda u+v, w)=\lambda f(u, w)+f(v, w), \\
& f(u, \lambda v+w)=\lambda f(u, v)+f(u, w) .
\end{aligned}
$$

The radical of $f$, denoted by $\operatorname{rad}(f)$, is $V^{\perp}=\{u \in V \mid f(u, v)=0, \forall v \in V\} . f$ is called non-singular if $\operatorname{rad}(f)=\langle 0\rangle$.

A map $Q: V \rightarrow K$ is called a quadratic form if it satisfies:

$$
Q(\lambda u+v)=\lambda^{2} Q(u)+\lambda f(u, v)+Q(v),
$$

where $f$ is a symmetric bilinear form. The radical of $Q$ is $\operatorname{rad}(Q)=\{v \in$ $\operatorname{rad}(f) \mid Q(v)=0\} . Q$ is called non-singular if $\operatorname{rad}(Q)=\langle 0\rangle$.

An isometry on a quadratic space $(V, Q)$ is a non-singular linear map $g$ : $V \rightarrow V$ such that $Q(g(v))=Q(v)$ for all $v \in V$. Two quadratic spaces $(V, Q)$ and $\left(V, Q^{\prime}\right)$ are said to be equivalent, if there is an isometry $g: V \rightarrow V$ [13,23]. Besides, the group of all isometries of an inner-product space $(V, f)$ is denoted by $\operatorname{Isom}(V, f)$ and that of all isometries of a quadratic space $(V, Q)$ is denoted by $\operatorname{Isom}(V, Q)$. When $q$ is a power of odd primes then we have, $\operatorname{Isom}(V, f)=$ $\operatorname{Isom}(V, Q)[13,23$.

A vector $v \in V$ is said to be isotropic if $f(v, v)=0$, singular if $Q(v)=0$ and non-singular if $Q(v) \neq 0$. A subspace $W$ of $V$ is called totally isotropic if $f(u, v)=0$ for all $u, v \in W$ and totally singular if $Q(v)=0$ for all $v \in W$. Besides, a point $\langle v\rangle \in P(V)$ is called a singular point if $v$ is a singular vector, and $\langle v\rangle$ is called an isotropic point in $P(V)$ if $v$ is an isotropic vector.

2.2 Logarithmic Signatures and Minimal Logarithmic Signatures for Finite Groups

Definition 1 (Logarithmic Signature) 8] Let $G$ be a finite group, $A \subseteq G$. Let $\alpha=\left[A_{1}, \cdots, A_{s}\right]$ be a sequence of ordered subsets $A_{i}$ of $G$ such that $A_{i}=\left[a_{i 1}, \cdots, a_{i r_{i}}\right]$ with $a_{i j} \in G\left(1 \leq j \leq r_{i}\right)$. Then $\alpha$ is called a logarithmic signature for $G$ (or $A$ ) if each $g \in G$ (or $A$ ) is uniquely represented as a product

$$
g=a_{1 j_{1}} \cdots a_{s j_{s}}
$$

with $a_{i j_{i}} \in A_{i}(1 \leq i \leq s)$. 
The sequences $A_{i}$ are called the blocks of $\alpha$, the length of $\alpha$ is defined to be $l(\alpha)=\sum_{i=1}^{s} r_{i}$. Let $|G|=\prod_{j=1}^{k} p_{j}^{a_{j}}$ (or $|A|=\prod_{j=1}^{k} p_{j}^{a_{j}}$ ) be the prime power decomposition of $|G|$ (or $|A|$ ) and $\alpha=\left[A_{1}, A_{2}, \ldots, A_{s}\right]$ be an LS for $G$ (or $A$ ). From [6], we have $l(\alpha) \geq \sum_{j=1}^{k} a_{j} p_{j}$.

Definition 2 (Minimal Logarithmic Signature) [8] A logarithmic signature $\alpha$ for a finite group $G$ (or $A$ ) with $l(\alpha)=\sum_{j=1}^{k} a_{j} p_{j}$ is called a minimal logarithmic signature (MLS) for $G$ (or $A$ ).

Lemma 1 [21] Let $A, B \leq G$, if $A$ and $B$ satisfy any one of the following two conditions:

(i) $G=A \times B$ is a direct product of $A$ and $B, A \cap B=\{1\}$

(ii) $G=A: B$ is a semi-direct product of $A$ and $B, A \cap B=\{1\}$.

Then, $[A, B]$ is an $L S$ for $G$.

Lemma 2 [21, 23] Let $H$ be a normal subgroup of $G, A \subseteq G$ and $\eta$ the canonical homomorphism $\eta: G \rightarrow G / H$ such that $a, b \in A, a \neq b$ imply that $a H \neq b H$. Let $A^{\prime}=\eta(A)$, and suppose hat $\left[A_{1}, A_{2}, \cdots, A_{k}\right]$ is an LS for $A$. Let $B_{i}=\eta\left(A_{i}\right) \subseteq G / H$ for $1 \leq i \leq k$. Then, $\left[B_{1}, B_{2}, \cdots, B_{k}\right]$ is an $L S$ for $A^{\prime}$.

Now, let $V$ be a finite dimensional vector space over $F_{q}, f$ be a bilinear form and $Q$ be a quadratic form. We call $L \subseteq P(V)$ a Singhi subset [23] if $L$ is one of the following sets [21,23]:

(i) the set of all isotropic points of $P(V)$ with respect to the bilinear form $f$,

(ii) the set of all non-isotropic points of $P(V)$ with respect to the bilinear form $f$

(iii) the set of all singular points of $P(V)$ with respect to the quadratic form $Q$

(iv) the set of all non-singular points of $P(V)$ with respect to the quadratic form $Q$.

(Note that a Singhi subset $L$ that meets condition (i) will be used in our later construction.)

Lemma 3 [21,23] Suppose that $G \mid L$ is a transitive permutation group action such that $G$ is a subgroup of $G L(V)$ and $L \subseteq P(V)$ is a Singhi subset. Let $S$ be an $r$-partial spread in $V$, which partitions $L$. Let $W \in S, w \in P(W)$ and $G_{w}$ be the stabilizer of $w$ in $G$. Suppose there are sets $A, B \subseteq G$ such that

(i) A acts sharply transitive on $S$ with respect to $W$ under the action of $G$ on the set of all $r$-dimensional subspaces of $V$.

(ii) $B$ acts sharply transitive on $L \cap P(W)$ with respect to $w$ under the action of $G$ on $P(W)$.

Then, $\left[A, B, G_{w}\right]$ is an $L S$ for $G$.

Lemma 4 [6, 21, 23] If $G$ is a solvable group, then $G$ has an $M L S$. 
Lemma 5 [23] Let $G$ be a finite group and $x \in G$ be an element of order $t$. For $s \in N, s \leq t$, let $S=\left\{x^{i} \mid 0 \leq i<s\right\}=\left\{1, x^{1}, x^{2}, \cdots, x^{s-1}\right\}$ be a cyclic set generated by $x$. Then $S$ has an $M L S \beta=\left[A_{1}, A_{2}, \cdots, A_{k}\right]$ satisfying the following condition:

For any list $\left[j_{i}, j_{2}, \cdots, j_{k}\right]$, such that $x^{j_{i}} \in A_{i}, 1 \leq i \leq k, \sum_{i=1}^{k} j_{i}<s$.

Lemma 6 23] Let $G$ be a finite group and $\left[A_{1}, \cdots, A_{r}\right]$ be an $L S$ for $G$ such that for each subset $A_{j}, 1 \leq j \leq r$, an $M L S$ exists. Then $G$ has an $M L S$.

\section{Construction I: LSs for Parabolic Subgroups of $O_{n}(q)$ and} $S O_{n}(q)$

Throughout this paper, we assume that $q$ is a power of odd primes. First, we construct the LSs for parabolic subgroups in $O_{2 m+1}(q)$ and $O_{2 m}^{ \pm}(q)$.

Let $W$ be an isotropic $k$-space of $V=F_{q^{2 m}}$, then the stabilizer of $W$ is the maximal parabolic subgroup $P_{\max }$ in $O_{2 m}^{ \pm}(q)$ of shape $q^{k(k-1) / 2+k(2 m-2 k)}$ : $\left(G L_{k}(q) \times O_{2 m-2 k}^{ \pm}(q)\right)$. Specifically, $P_{\max }$ has the shape

$$
\left(\begin{array}{ccccc}
1 & 0 & 0 & \cdots & 0 \\
\vdots & \ddots & \vdots & & \vdots \\
* & \cdots & 1 & \cdots & 0 \\
0 & 0 & 0 & A & 0 \\
0 & 0 & 0 & C & D
\end{array}\right)
$$

the normal $p$-subgroup $R$ is a group of shape

$$
\left(\begin{array}{ccccc}
1 & 0 & 0 & \cdots & 0 \\
\vdots & \ddots & \vdots & & \vdots \\
* & \cdots & 1 & \cdots & 0 \\
0 & 0 & 0 & I_{k} & 0 \\
0 & 0 & 0 & C^{\prime} & I_{2 m-2 k}
\end{array}\right)
$$

with center of order $q^{k(k-1) / 2}$ and the subset $Q$ of matrices of the shape

$$
\left(\begin{array}{ccc}
I_{k} & 0 & 0 \\
0 & A & 0 \\
0 & 0 & D
\end{array}\right)
$$

is a subgroup isomorphic to $G L_{k}(q) \times O_{2 m-2 k}^{ \pm}(q)$. Moreover, $R \cap Q=\left\{I_{n}\right\}$, $P_{\max }=R: Q$, therefore, $P_{\max }$ has an LS $[R, Q]$ (see Lemma 1). Also, $P_{\max }$ is of shape $q^{k(k-1) / 2+k(2 m+1-2 k)}:\left(G L_{k}(q) \times O_{2 m+1-2 k}(q)\right)$ in $O_{2 m+1}(q)$.

Similarly, for $S O_{2 m}^{ \pm}(q)$ and $S O_{2 m+1}(q), P_{\text {max }}^{\prime}$ is of shape $q^{k(k-1) / 2+k(2 m-2 k)}$ : $\left(G L_{k}(q) \times S O_{2 m-2 k}^{ \pm}(q)\right)$ in $S O_{2 m}^{ \pm}(q)$ and $q^{k(k-1) / 2+k(2 m+1-2 k)}:\left(G L_{k}(q) \times\right.$ $\left.S O_{2 m+1-2 k}(q)\right)$ in $S O_{2 m+1}(q)$. 


\section{Construction II: MLSs for $O_{2 m}^{-}(q)$ and $S O_{2 m}^{-}(q)$}

Now, we construct MLSs for $\mathrm{O}_{2 m}^{-}(q)$ and $\mathrm{SO}_{2 m}^{-}(q)$. Here, our fundamental tools are Lemma 3 and Lemma 6. Choosing suitable quadratic form $Q$ of minus type, we take advantage of all isotropic points of $P(V)$ for constructing the corresponding MLSs.

First, we observe $O_{2 m}^{-}(q)$. Suppose $q$ is a power of odd primes, $V=F_{q^{2 m}}$ is a $2 m$-dimensional vector space over $F_{q}$, and $L$ is the set of all isotropic points of $P(V)$. For $y \in V, \bar{y}$ denotes $y^{q^{m}} . T_{s}: V \rightarrow V$ is a linear transformation defined by $T_{s}(v)=s v$ for a given $s \in V$ and all $v \in V$. Let $\alpha$ be a primitive element of the field $F_{q^{2 m}}$ and $x \in G L_{2 m}(q)$ be the matrix corresponding to the linear transformation $T_{\alpha}$ [23]. We define a bilinear map $f: V \times V \rightarrow F_{q}$ by $f(x, y)=t r_{F_{q} m / F_{q}}(x \bar{y}+\bar{x} y)=\sum_{i=0}^{m-1}(x \bar{y}+\bar{x} y)^{q^{i}}$ and a quadratic form $Q: V \rightarrow F_{q}$ by $Q(x)=t r_{F_{q} / F_{q}}(x \bar{x})=\sum_{i=0}^{m-1}(x \bar{x})^{q^{i}}[21,23]$. Then, we observe that the number of non-zero isotropic points in $P(V)$ with respect to the quadratic space $(V, Q)$ are $\left(q^{m}+1\right)\left(q^{m-1}-1\right) /(q-1)$ and the quadratic form $Q$ is of minus type 13,23 . Let $G$ be the group of all isometries of $(V, Q)$, then $G \cong O_{2 m}^{-}(q)$ and $G$ is a permutation group acting transitively on isotropic points 13,23 .

Now, we roughly explain the main idea for constructing the MLSs. As described above, since the number of non-zero isotropic points in $P(V)$ with respect to the quadratic space $(V, Q)$ are $\left(q^{m}+1\right)\left(q^{m-1}-1\right) /(q-1)$, therefore, we need to construct a cyclic group $A$ of order $q^{m}+1$, which must be sharply transitive on a partial spread $S$ and a cyclic set $B$ of cardinality $\left(q^{m-1}-\right.$ $1) /(q-1)$, which must be sharply transitive on the projective subspace of $P(V)$. Then, we take advantage of the Lemma 3. Lemma 5 and Lemma 6 for proving the existence of MLSs for $O_{2 m}^{-}(q)$.

First, we define two special cyclic subgroups of $O_{2 m}^{-}(q)$. Let $a_{1}=x_{1}^{q^{m}-1} \in$ $G L_{2 m}(q)$ be the matrix corresponding to the linear transformation $T_{\alpha^{q^{m}}-1}$. Let $W_{1}^{\prime}=\left\{e_{1}, e_{2}, \cdots, e_{m-1}\right\}$ is an $(m-1)$-dimensional totally isotropic subspace of $V, D_{1} \in G L\left(W_{1}^{\prime}\right)$ be a generator of the Singer cyclic subgroup of $G L\left(W_{1}^{\prime}\right)$ [19]. Then $b_{1} \in G L_{2 m}(q)$ can be well defined as follows:

$$
b_{1}=\left(\begin{array}{cccc}
D_{1} & 0 & 0 & 0 \\
0 & 1 & 0 & 0 \\
0 & 0 & \left(D_{1}^{t}\right)^{-1} & 0 \\
0 & 0 & 0 & 1
\end{array}\right)
$$

Meanwhile, we get that $a_{1}, b_{1} \in G\left[1,23\right.$. Let $A_{1}=\left\langle a_{1}\right\rangle, B_{1}=\left\langle b_{1}\right\rangle$ be the cyclic subgroups of $G$ generated by $a_{1}$ and $b_{1}$, respectively. Then, $A_{1}$ is of order $q^{m}+1$ and $B_{1}$ is of order $q^{m-1}-1$.

Let $C_{1}=\left\langle b_{1}^{\frac{q^{m-1}-1}{q-1}}\right\rangle$ be the subgroup of order $q-1$ of $B_{1}$ and $B_{1}^{\prime}=\left\{g C_{1} \mid g \in\right.$ $\left.B_{1}\right\}$ be the left coset of $C_{1}$ in $B_{1}$. Thus, $\left|B_{1}^{\prime}\right|=\frac{q^{m}-1}{q-1}$. Furthermore, $A_{1}$ and $B_{1}^{\prime}$ are chosen so that $A_{1} \cap B_{1}^{\prime}=\{1\}$ and both are cyclic sets. Then, from Lemma 5. it follows that $A_{1}$ and $B_{1}^{\prime}$ have MLSs. 
Now, let $S_{1}^{\prime}=\left\{W_{i}^{\prime} \mid 0 \leq i \leq q^{m}\right\}$ be the classical spread as described in Remark 1. $W_{i}^{\prime}$ are $(m-1)$-dimensional totally isotropic subspaces of $V$ for $0 \leq i \leq q^{m}$. Clearly, the partial spread $S_{1}^{\prime}$ partitions the set of all isotropic points of $P(V)$.

Also, we observe that the group $A_{1}$ is sharply transitive on $S_{1}^{\prime}$ with respect to $W_{1}^{\prime}$. Also, it is clear that $B_{1}$ is isomorphic to the Singer cyclic subgroup of $G L_{m-1}(q)$ and $B_{1}^{\prime}$ is sharply transitive on $P\left(W_{1}^{\prime}\right)$ with respect to $\left\langle e_{1}\right\rangle$, where $e_{1} \in W_{1}^{\prime}$.

Now, we consider $G^{*}=S O_{2 m}^{-}(q)$. Being similar to the case of $O_{2 m}^{-}(q)$, let $a_{1}^{*}=x_{1}^{* q^{m}-1} \in S O_{2 m}^{-}(q)$ be the matrix corresponding to the linear transformation $T_{\alpha^{q}{ }^{m}-1}$. Let $W_{1}^{\prime}=\left\{e_{1}, e_{2}, \cdots, e_{m-1}\right\}$ is an $(m-1)$-dimensional totally isotropic subspace of $V$. Let $D_{1}^{*} \in O_{m-1}(q) \leq G L\left(W_{1}^{\prime}\right), b_{1}^{*} \in S O_{2 m}^{-}(q)$ is presented well defined as follows:

$$
b_{1}^{*}=\left(\begin{array}{cccc}
D_{1}^{*} & 0 & 0 & 0 \\
0 & 1 & 0 & 0 \\
0 & 0 & D_{1}^{* t} & 0 \\
0 & 0 & 0 & 1
\end{array}\right)
$$

thus, $A_{1}^{*}=\left\langle a_{1}^{*}\right\rangle$, and $B_{1}^{*}=\left\langle b_{1}^{*}\right\rangle$ are the cyclic subgroups of $S O_{2 m}^{-}(q)$ generated by $a_{1}^{*}$ and $b_{1}^{*}$ with order $q^{m}+1$ and $q^{m-1}-1$, respectively.

Let $C_{1}^{*}=\left\langle b_{1}^{* \frac{q^{m-1}-1}{q-1}}\right\rangle$ be the subgroup of order $q-1$ of $B_{1}^{*}$ and $B_{1}^{\prime *}=$ $\left\{g C_{1}^{*} \mid g \in B_{1}^{*}\right\}$ be the left coset of $C_{1}^{*}$ in $B_{1}^{*}$. Thus, $\left|B_{1}^{\prime *}\right|=\frac{q^{m}-1}{q-1}$. Furthermore, the cyclic sets $A_{1}^{*}$ and $B_{1}^{\prime *}$ are chosen so that $A_{1}^{*} \cap B_{1}^{\prime *}=\{1\}$. Consequently, from Lemma 5 it follows that $A_{1}^{*}$ and $B_{1}^{\prime *}$ have MLSs.

Also, let $S_{1}^{\prime}=\left\{W_{i}^{\prime} \mid 0 \leq i \leq q^{m}\right\}$ be the classical spread as described in Remark 1 $W_{i}^{\prime}$ are $(m-1)$-dimensional totally isotropic subspaces of $V$ for $0 \leq i \leq q^{m}$. It's clear that the partial spread $S_{1}^{\prime}$ partitions the set of all isotropic points of $P(V)$.

We observe that the group $A_{1}^{*}$ is sharply transitive on $S_{1}^{\prime}$ with respect to $W_{1}^{\prime}$. Also, $B_{1}^{*}$ is isomorphic to the Singer cyclic subgroup of $G L_{m-1}(q)$ and $B_{1}^{\prime *}$ is sharply transitive on $P\left(W_{1}^{\prime}\right)$ with respect to $\left\langle e_{1}\right\rangle$, where $e_{1} \in W_{1}^{\prime}$. Hence, we have the following lemma from the fact above.

Lemma 7 Let $A_{1}, B_{1}^{\prime} \subseteq O_{2 m}^{-}(q)$ (resp. $A_{1}^{*}, B_{1}^{\prime *} \subseteq S O_{2 m}^{-}(q)$ ), $S_{1}^{\prime}$ be the partial spread, $W_{1}^{\prime}$ be the subspace of $V$ and $w_{1}=\left\langle e_{1}\right\rangle$. Then,

(i) $A_{1}$ (resp. $\left.A_{1}^{*}\right)$ is a sharply transitive set on $S_{1}^{\prime}$ with respect to $W_{1}^{\prime}$.

(ii) $B_{1}^{\prime}\left(\right.$ resp. $\left.B_{1}^{\prime *}\right)$ is a sharply transitive set on $P\left(W_{1}^{\prime}\right)$ with respect to $w_{1}$.

Theorem 1 Let $q$ be a power of odd primes. Then, the orthogonal group $\mathrm{O}_{2 m}^{-}(q)$ has an $M L S$.

Proof In case, when $m=1, G=O_{2}^{-}(q) \cong D_{q+1}$ which is a dihedral group of order 2(q+1). From Lemma $4 . O_{2}^{-}(q)$ has an MLS. When $m>1$, let $A=A_{1}$, $B=B_{1}^{\prime}, w_{1}=\left\langle e_{1}\right\rangle, L$ be the set of all isotropic points of $P(V)$. Then, from Lemma 3 and Lemma [7 $\left[A_{1}, B_{1}^{\prime}, G_{w}\right]$ is an LS for $G$. The stabilizer $G_{w}$ is 
a semi-direct product of a $p$-group of order $q^{2 m-2}$ and $G L_{1}(q) \times O_{2 m-2}^{-}(q)$. Now from Lemma 4 , groups and $G L_{1}(q)$ have MLSs. Furthermore, by the induction hypothesis, we assume that $O_{2 m-2}^{-}(q)$ has an MLS. Thus, $G_{w}$ has an MLS. Also, from Lemma 5. $A_{1}$ and $B_{1}^{\prime}$ have MLSs. Hence, using Lemma 6. $G$ has an MLS.

Theorem 2 Let $q$ be a power of odd primes. Then, the special orthogonal group $S O_{2 m}^{-}(q)$ has an $M L S$.

Proof In case, when $m=1, G^{*}=S O_{2}^{-}(q)$ is a solvable group of order $q+1$. Lemma 4 implies that $S O_{2}^{-}(q)$ has an MLS. When $m>1$, let $A=A_{1}^{*}, B=$ $B *_{1}^{\prime}, w_{1}=\left\langle e_{1}\right\rangle, L$ be the set of all isotropic points of $P(V)$. Thus, from Lemma 3 and Lemma [7. $\left[A_{1}^{*}, B_{1}^{\prime *}, G_{w}^{*}\right]$ is an LS for $S O_{2 m}^{-}(q)$. The stabilizer $G_{w}^{*}$ is a semi-direct product of a $p$-group of order $q^{2 m-2}$ and $G L_{1}(q) \times S O_{2 m-2}^{-}(q)$. Now, from Lemma 4, $p$-groups and $G L_{1}(q)$ have MLSs. Furthermore, by the induction hypothesis, we assume that $S O_{2 m-2}^{-}(q)$ has an MLS. Thus, $G_{w}^{*}$ has an MLS. Also, from Lemma 5, $A_{1}^{*}$ and $B_{1}^{\prime *}$ have MLSs. Hence, using Lemma 6] $S O_{2 m}^{-}(q)$ has an MLS.

\section{Construction III: MLSs for $O_{2 m}^{+}(q)$ and $S O_{2 m}^{+}(q)$}

Similarly, we construct MLSs for $O_{2 m}^{+}(q)$ and $S O_{2 m}^{+}(q)$. Also, $L$ is the set of all isotropic points of $P(V)$.

We at first consider $O_{2 m}^{+}(q)$. Let $V=F_{q^{2 m}}, \alpha$ a primitive element of field $F_{q^{2 m}}$ and $q$ be a power of odd primes. The corresponding bilinear map and quadratic form are described as $f(x, y)=f\left(x_{1}+x_{2} \beta, y_{1}+y_{2} \beta\right)=t r_{F_{q} / F_{q}}\left(x_{1} y_{2}+\right.$ $\left.x_{2} y_{1}\right)=\sum_{i=0}^{m-1}\left(x_{1} y_{2}+x_{2} y_{1}\right)^{q^{i}}$ and $Q(x)=Q\left(x_{1}+x_{2} \beta\right)=\operatorname{tr}_{F_{q} / F_{q}}\left(x_{1} x_{2}\right)=$ $\sum_{i=0}^{m-1}\left(x_{1} x_{2}\right)^{q^{i}}$, respectively, where $\beta=\alpha^{q^{m}-1}$ [23]. We observe that the number of non-zero isotropic points in $P(V)$ with respect to the quadratic space $(V, Q)$ are $\left(q^{m}-1\right)\left(q^{m-1}+1\right) /(q-1)$ and the quadratic form $Q$ is of plus type $\left[13,23\right.$. Let $G$ be the group of all isometries of $(V, Q)$, then, $G \cong O_{2 m}^{+}(q)$ and $G$ is a permutation group acting transitively on isotropic points [13, 23.

Similarly, we must have to construct a cyclic group $A$ of order $q^{m-1}+$ 1 which is sharply transitive on a partial spread $S$ and a cyclic set $B$ of cardinality $\left(q^{m}-1\right) /(q-1)$ which is sharply transitive on $P(W)$, the projective subspace of $P(V)$. Furthermore, we also need to use the Lemma 3 , Lemma 5 and Lemma 6 to prove the existence of MLSs.

First, we define two special cyclic subgroups of $O_{2 m}^{+}(q)$. Let $a_{2}=x_{2}^{q^{m-1}-1} \in$ $G L_{2 m}(q)$ be the matrix corresponding to the linear transformation $T_{\alpha^{q^{m-1}-1}}$, where $x_{2}=\left(\begin{array}{lll}x & 0 & 0 \\ 0 & 1 & 0 \\ 0 & 0 & 1\end{array}\right), x \in G L_{2 m-2}(q)$. Let $W_{2}=\left\{e_{1}, e_{2}, \cdots, e_{m}\right\}$ be an $m$ dimensional totally isotropic subspace of $V, D_{2} \in G L\left(W_{2}\right)$ be a generator of the Singer cyclic subgroup of $G L\left(W_{2}\right)$. Then, $b_{2} \in G L_{2 m}(q)$ is defined as follows: [1,23. 


$$
b_{2}=\left(\begin{array}{cc}
D_{2} & 0 \\
0 & \left(D_{2}^{t}\right)^{-1}
\end{array}\right)
$$

Meanwhile, we have that $a_{2}, b_{2} \in G\left[1,23\right.$. Let $A_{2}=\left\langle a_{2}\right\rangle$ and $B_{2}=\left\langle b_{2}\right\rangle$ be the cyclic subgroups of $G$ generated by $a_{2}$ and $b_{2}$, respectively. Then, $A_{2}$ is of order $q^{m-1}+1$ and $B_{2}$ is of order $q^{m}-1$.

Let $C_{2}=\left\langle b_{2}^{\frac{q^{m}-1}{q-1}}\right\rangle$ be the subgroup of order $q-1$ of $B_{2}$ and $B_{2}^{\prime}=\left\{g C_{2} \mid g \in\right.$ $\left.B_{2}\right\}$ be the left coset of $C_{2}$ in $B_{2}$. Thus, $\left|B_{2}^{\prime}\right|=\frac{q^{m}-1}{q-1}$. Furthermore, the cyclic sets $A_{2}$ and $B_{2}^{\prime}$ are chosen so that $A_{2} \cap B_{2}^{\prime}=\{1\}$. Then from Lemma [5, it follows that $A_{2}$ and $B_{2}^{\prime}$ have MLSs.

Now, let $S_{2}=\left\{W_{i} \mid 0 \leq i \leq q^{m}\right\}$ be the classical spread as described in Remark 1. $W_{i}$ are m-dimensional totally isotropic subspaces of $V$ for $0 \leq i \leq$ $q^{m}$. Then, the partial spread $S_{2}$ clearly partitions the set of all isotropic points of $P(V)$.

Also, we observe that the group $A_{2}$ is sharply transitive on $S_{2}$ with respect to $W_{2}$. Also, it is clear that $B_{2}^{\prime}$ is sharply transitive on $P\left(W_{2}\right)$ with respect to $\left\langle e_{1}\right\rangle$, where $e_{1} \in W_{2}$.

Now, we take account for $G^{*}=S O_{2 m}^{+}(q)$. Being similar to $O_{2 m}^{+}(q), a_{2}^{*}=$ $x_{2}^{* q^{m-1}-1} \in S O_{2 m}^{+}(q)$ is also the matrix corresponding to the linear transforma$\operatorname{tion} T_{\alpha^{q^{m-1}-1}}$, where $x_{2}^{*}=\left(\begin{array}{ccc}x^{*} & 0 & 0 \\ 0 & 1 & 0 \\ 0 & 0 & 1\end{array}\right), x^{*} \in S O_{2 m-2}^{+}(q)$. Let $W_{2}=\left\{e_{1}, e_{2}, \cdots, e_{m}\right\}$ be an $m$-dimensional totally isotropic subspace of $V$ and $D_{2}^{*} \in G L\left(W_{2}\right)$ be a generator of the Singer cyclic subgroup of $G L\left(W_{2}\right)$. Then $b_{2}^{*} \in S O_{2 m}^{+}(q)$ is defined as follows: 23

$$
b_{2}^{*}=\left(\begin{array}{cc}
D_{2}^{*} & 0 \\
0 & D_{2}^{* t}
\end{array}\right)
$$

Hence, $A_{2}=\left\langle a_{2}\right\rangle$ and $B_{2}=\left\langle b_{2}\right\rangle$ are the cyclic subgroups of $G$ with order $q^{m-1}+1$ and $q^{m}-1$, respectively.

Let $C_{2}^{*}=\left\langle b_{2}^{* q^{m}-1} \frac{1}{q-1}\right\rangle$ be the subgroup of order $q-1$ of $B_{2}^{*}$ and $B_{2}^{*}=\left\{g C_{2}^{*} \mid g \in\right.$ $\left.B_{2}^{*}\right\}$ be the left coset of $C_{2}^{*}$ in $B_{2}^{*}$. Thus, $\left|B_{2}^{* *}\right|=\frac{q^{m}-1}{q-1}$. Furthermore, the cyclic sets $A_{2}^{*}$ and $B_{2}^{\prime *}$ are chosen so that $A_{2}^{*} \cap B_{2}^{\prime *}=\{1\}$. Then from Lemma 5 , it follows that $A_{2}^{*}$ and $B_{2}^{\prime *}$ have MLSs.

Now, let $S_{2}=\left\{W_{i} \mid 0 \leq i \leq q^{m}\right\}$ the classical spread as described in Remark 1. $W_{i}$ are m-dimensional totally isotropic subspaces of $V$ for $0 \leq i \leq$ $q^{m}$. Thus, the partial spread $S_{2}$ clearly partitions the set of all isotropic points of $P(V)$.

Consequently, we observe that the group $A_{2}^{*}$ is sharply transitive on $S_{2}$ with respect to $W_{2}$. Also, it is clear that $B_{2}^{\prime *}$ is sharply transitive on $P\left(W_{2}\right)$ with respect to $\left\langle e_{1}\right\rangle$, where $e_{1} \in W_{2}$. Hence, we have the following lemma.

Lemma 8 Let $A_{2}, B_{2}^{\prime} \subseteq O_{2 m}^{+}(q)$ (resp. $A_{2}^{*}, B_{2}^{\prime *} \subseteq S O_{2 m}^{+}(q)$ ), $S_{2}$ be the partial spread, $W_{2}$ be the subspace of $V$, and $w_{2}=\left\langle e_{1}\right\rangle$. Then,

(i) $A_{2}$ (resp. $A_{2}^{*}$ ) is a sharply transitive set on $S_{2}$ with respect to $W_{2}$. 
(ii) $B_{2}^{\prime}$ (resp. $\left.B_{2}^{\prime *}\right)$ is a sharply transitive set on $P\left(W_{2}\right)$ with respect to $w_{2}$.

Theorem 3 Let $q$ be a power of odd primes. Then, the orthogonal group $\mathrm{O}_{2 m}^{+}(q)$ has an $M L S$.

Proof Let $G=O_{2 m}^{+}(q)$. In case,when $m=1, O_{2}^{+}(q)$ is of order $2(q-1)$. Then, by using Lemma 4, $O_{2}^{+}(q)$ has an MLS. When $m>1$, let $A=A_{2}, B=B_{2}^{\prime}$, $w_{2}=\left\langle e_{1}\right\rangle, L$ be the set of all isotropic points of $P(V)$. Hence, from Lemma 5 and Lemma 8, $\left[A_{2}, B_{2}^{\prime}, G_{w_{2}}\right]$ is an LS for $G$. Besides, the stabilizer $G_{w_{2}}$ is a semi-direct product of a $p$-group of order $q^{2 m-2}$ and $G L_{1}(q) \times O_{2 m-2}^{+}(q)$. Now from Lemma 4, $p$-groups and $G L_{1}(q)$ have MLSs. Furthermore, by the induction hypothesis, we assume that $O_{2 m-2}^{+}(q)$ has an MLS, therefore, $O_{2 m}^{+}(q)$ also has an MLS. Thus, $G_{w_{2}}$ has an MLS. Also, from Lemma 5 , the cyclic sets $A_{2}$ and $B_{2}^{\prime}$ have MLSs. Therefore, using Lemma 6. $G$ has an MLS.

Theorem 4 Let $q$ be a power of odd primes. Then, special orthogonal group $S O_{2 m}^{+}(q)$ has an $M L S$.

Proof In case, when $n=1, G^{*}=S O_{2}^{+}(q)$ is a solvable group of order $q-1$. Then by using Lemma 4, $S O_{2}^{+}(q)$ has an MLS. When $m>1$, let $A=A_{2}^{*}, B=$ $B_{2}^{\prime *}, w_{2}=\left\langle e_{1}\right\rangle, L$ be the set of all isotropic points of $P(V)$. Hence, from Lemma [5] and Lemma 8, $\left[A_{2}^{*}, B_{2}^{\prime *}, G_{w_{2}}^{*}\right]$ is an LS for $G$. Besides, the stabilizer $G_{w_{2}}^{*}$ is a semi-direct product of a $p$-group of order $q^{2 m-2}$ and $G L_{1}(q) \times S O_{2 m-2}^{+}(q)$. Now from Lemma 4, $p$-groups and $G L_{1}(q)$ have MLSs. Furthermore, by the induction hypothesis, we assume that $S O_{2 m-2}^{+}(q)$ has an MLS, therefore, $G_{w_{2}}^{*}$ has an MLS. Also, from Lemma 5, the cyclic sets $A_{2}^{*}$ and $B_{2}^{\prime *}$ have MLSs. Finally, using Lemma 6, $S O_{2 m}^{+}(q)$ has an MLS.

\section{Construction IV: MLSs for $P S O_{2 m}^{ \pm}(q)$ and $P \Omega_{2 m}^{ \pm}(q)$}

In this section, we consider the MLSs for $P S O_{2 m}^{ \pm}(q)$ and $P \Omega_{2 m}^{ \pm}(q)$. Being different from $O_{2 m}^{ \pm}(q)$ and $S O_{2 m}^{ \pm}(q)$, our technique is based on some canonical homomorphisms.

Theorem 5 Let $q$ be a power of odd primes. Then, $P S O_{2 m}^{ \pm}(q)$ has an $M L S$.

Proof (1)In case, when $G^{*}=S O_{2 m}^{-}(q)$ and $G^{\prime}=P S O_{2 m}^{-}(q)$, let $A=A_{1}^{*}$, $B=B_{1}^{\prime *}, w_{1}=\left\langle e_{1}\right\rangle, L$ be the set of all isotropic points of $P(V)$ as described as Section 3. Suppose $\eta_{1}: S O_{2 m}^{-}(q) \rightarrow P S O_{2 m}^{-}(q) \cong S O_{2 m}^{-}(q) / Z\left(S O_{2 m}^{-}(q)\right.$ is the canonical homomorphism onto $P S O_{2 m}^{-}(q)$, and let $\overline{A_{1}^{*}}=\eta\left(A_{1}^{*}\right), \overline{B_{1}^{\prime *}}=\eta\left(B_{1}^{\prime *}\right)$ and $\overline{G_{w_{1}}^{*}}=\eta\left(G_{w_{1}}^{*}\right)$, then $\left[\overline{A_{1}^{*}}, \overline{B_{1}^{\prime *}}, \overline{G_{w_{1}}^{*}}\right]$ is the corresponding LS for $P S O_{2 m}^{-}(q)$ from Lemma 2, Also from Section 3 , the stabilizer $\overline{G_{w_{1}}^{*}}$ is a semi-direct product of a $p$-group of order $q^{2 m-2}$ and $G L_{1}(q) \times P S O_{2 m-2}^{-}(q)$. Thus, using the same induction as used in Theorem 2, we get that $P S O_{2 m}^{-}(q)$ has an MLS.

(2)In case, when $G^{*}=S O_{2 m}^{+}(q)$ and $G^{\prime}=P S O_{2 m}^{+}(q)$, let $A=A_{2}^{*}, B=$ $B_{2}^{\prime *}, w_{2}=\left\langle e_{1}\right\rangle, L$ be the set of all isotropic points of $P(V)$ as described as 
Section 4. Suppose $\eta_{2}: S O_{2 m}^{+}(q) \rightarrow P S O_{2 m}^{+}(q) \cong S O_{2 m}^{+}(q) / Z\left(S O_{2 m}^{+}(q)\right.$ is the canonical homomorphism onto $P S O_{2 m}^{+}(q)$, and let $\overline{A_{2}^{*}}=\eta\left(A_{2}^{*}\right), \overline{B_{2}^{\prime *}}=\eta\left(B_{2}^{\prime *}\right)$ and $\overline{G_{w_{2}}^{*}}=\eta\left(G_{w_{2}}^{*}\right)$, then $\left[\overline{A_{2}^{*}}, \overline{B_{2}^{\prime *}}, \overline{G_{w_{2}}^{*}}\right]$ is the corresponding LS for $P S O_{2 m}^{+}(q)$ from Lemma 2. Hence, the stabilizer $\overline{G_{w_{2}}^{*}}$ is a semi-direct product of a $p$-group of order $q^{2 m-2}$ and $G L_{1}(q) \times P S O_{2 m-2}^{+}(q)$. Thus, using the same induction as used in Theorem 2, we get that $P S O_{2 m}^{+}(q)$ has an MLS.

Theorem 6 Let $q$ be a power of odd primes. Then, $P \Omega_{2 m}^{ \pm}(q)$ has an $M L S$.

Proof (1)In case, when $q^{m} \equiv-1 \bmod 4$, from [13, we have that $P S O_{2 m}^{ \pm}(q)=$ $P \Omega_{2 m}^{ \pm}(q)$. Therefore, being similar to the case in $P S O_{2 m}^{ \pm}(q),\left[{\overline{A_{1}^{*}}}_{B_{1}^{\prime *}}, \bar{G}_{w_{1}}^{*}\right]$ is the corresponding LS for $P \Omega_{2 m}^{-}(q)$ and $\left[\overline{A_{2}^{*}}, \overline{B_{2}^{\prime *}}, \overline{G_{w_{2}}^{*}}\right]$ is the corresponding LS for $P \Omega_{2 m}^{+}(q)$. Meanwhile, as described in Theorm [5, $P \Omega_{2 m}^{-}(q)$ and $P \Omega_{2 m}^{+}(q)$ both have MLSs.

(2)In case, when $q^{m} \equiv 1 \bmod 4$, we must consider the reflection in $G^{*}=$ $S O_{n}(q)$. For the isotropic 1-space $w=\left\langle e_{1}\right\rangle$, the reflection $r_{w}: V \rightarrow V$ is defined by $r_{w}(v)=v-2 \frac{f(v, w)}{f(w, w)} w$ for each $v \in V$. Also, the linear transformation $r_{w}$ is an element of $G_{w}^{*}$. For $G^{\prime}=\Omega_{2 m}^{-}(q)$ (resp. $\Omega_{2 m}^{+}(q)$ ), each element of $G_{w}^{\prime}$ is a product of an even number of reflections and $G_{w}^{\prime}$ is a semi-direct product of a $p$-group of order $q^{2 m-2}$ and $G L_{1}(q) \times \Omega_{2 m-2}^{-}(q)\left(\operatorname{resp} . G L_{1}(q) \times \Omega_{2 m-2}^{+}(q)\right)$. Besides, an element $x$ in $S O_{2 m}^{ \pm}(q)$ is in $\Omega_{2 m}^{ \pm}(q)$ if and only if the rank of $I_{2 m}+x$ is even. From the construction of $A_{1}^{*}$ (resp. $A_{2}^{*}$ ) and $B_{1}^{\prime *}$ (resp. $B_{2}^{\prime *}$ ) in Section 3 and Section 4, we get that the ranks of $I_{2 m}+x_{1}^{*}\left(\right.$ resp. $\left.I_{2 m}+x_{2}^{*}\right)$ and $I_{2 m}+b_{1}^{*}$ (resp. $\left.I_{2 m}+b_{2}^{*}\right)$ are both even, Thus, $A_{1}^{*}\left(\right.$ resp. $\left.A_{2}^{*}\right) \leq G^{\prime}, B_{1}^{\prime *}$ (resp. $\left.B_{2}^{\prime *}\right) \subseteq G^{\prime}$. As described in Theorem 3 and Theorem 4 , we have $\Omega_{2 m}^{-}(q)$ (resp. $\left.\Omega_{2 m}^{+}(q)\right)$ has an MLS.

Furthermore, let $\theta_{1}: \Omega_{2 m}^{-}(q) \rightarrow P \Omega_{2 m}^{-}(q)$ and $\theta_{2}: \Omega_{2 m}^{+}(q) \rightarrow P \Omega_{2 m}^{+}(q)$ be the canonical homomorphisms onto $P \Omega_{2 m}^{-}(q)$ and $P \Omega_{2 m}^{+}(q)$, respectively. Therefore, $\left[\theta_{1}\left(A_{1}^{*}\right), \theta_{1}\left(B_{1}^{\prime *}\right), \theta_{1}\left(G_{w_{1}}^{*}\right)\right]$ is the corresponding LS for $P \Omega_{2 m}^{-}(q)$ and $\left[\theta_{2}\left(A_{2}^{*}\right), \theta_{2}\left(B_{2}^{\prime *}\right), \theta_{2}\left(G_{w_{2}}^{*}\right)\right]$ is the corresponding LS for $P \Omega_{2 m}^{+}(q)$. Also, for $P \Omega_{2 m}^{-}(q)$, the stabilizer $\theta_{1}\left(G_{w_{1}}^{*}\right)$ is a semi-direct product of a $p$-group of order $q^{2 m-2}$ and $G L_{1}(q) \times P \Omega_{2 m-2}^{-}(q)$; for $P \Omega_{2 m}^{+}(q)$. the stabilizer $\theta_{2}\left(G_{w_{2}}^{*}\right)$ is a semi-direct product of a $p$-group of order $q^{2 m-2}$ and $G L_{1}(q) \times P \Omega_{2 m-2}^{+}(q)$. Thus, using the same induction as used in Theorem 2 and Theorem 4 , we get that $P \Omega_{2 m}^{-}(q)$ and $P \Omega_{2 m}^{+}(q)$ both have MLSs.

\section{Construction V: MLSs for $O_{2 m+1}(q), S O_{2 m+1}(q), P S O_{2 m+1}(q)$ and $P \Omega_{2 m+1}(q)$}

In this section, we first construct MLSs for $O_{2 m+1}(q)$ and $S O_{2 m+1}(q)$. Then we consider the MLSs for $P S O_{2 m+1}(q)$ and $P \Omega_{2 m+1}(q)$. Here, $L$ is also the set of all isotropic points of $P(V)$.

Let $V=F_{q^{2 m+1}}$ and $q$ be a power of odd primes. The corresponding nonsingular alternating bilinear map is $f(x, y)=\operatorname{tr}_{F_{q^{2 m+1}} / F_{q}}(a x \bar{y})=\sum_{i=1}^{2 m+1}(a x \bar{y})^{q^{i}}$, 
where $a \in F_{q^{2 m+1}}^{*}$ and $a+\bar{a}=0$ [23. Also, $G=O_{2 m+1}(q)$ is the isometry group of the inner product space $(V, f)$ and $G$ is a permutation group acting transitively on isotropic points [13,23. Then we observe that the number of non-zero isotropic points in $P(V)$ with respect to the inner product space $(V, f)$ are $\left(q^{m}-1\right)\left(q^{m}+1\right) /(q-1)$ [13.23.

Similarly, we construct a cyclic group $A$ of order $q^{m}+1$, which is sharply transitive on a partial spread $S$ and a cyclic set $B$ of cardinality $q^{m}-1 /(q-1)$, which is sharply transitive on $P(W)$, the projective subspace of $P(V)$. Then we use the Lemma 3. Lemma 5 and Lemma 6 to prove the existence of MLSs.

Let $a_{3}=x_{3}^{q^{m}-1} \in G L_{2 m+1}(q)$ be the matrix corresponding to the linear transformation $T_{\alpha^{q^{m}-1}}$, where $x_{3}=\left(\begin{array}{ll}x & 0 \\ 0 & 1\end{array}\right), x \in G L_{2 m}(q)$. Let $W_{3}=$ $\left\{e_{1}, e_{2}, \cdots, e_{m}\right\}$ be an $m$-dimensional totally isotropic subspace of $V, D_{3} \in$ $G L\left(W_{3}\right)$ be a generator of the Singer cyclic subgroup of $G L\left(W_{3}\right)$. Then $b_{3} \in$ $G L_{2 m+1}(q)$ defined as follows: 23 .

$$
b_{3}=\left(\begin{array}{ccc}
D_{3} & 0 & 0 \\
0 & \left(D_{3}^{t}\right)^{-1} & 0 \\
0 & 0 & 1
\end{array}\right)
$$

From [1,23], we have that $a_{3}, b_{3} \in G$. Let $A_{3}=\left\langle a_{3}\right\rangle, B_{3}=\left\langle b_{3}\right\rangle$ be cyclic subgroups of $G$. Thus, $\left|A_{3}\right|=q^{m}+1$ and $\left|B_{3}\right|=q^{m}-1$.

Let $C_{3}=\left\langle b_{3}^{\frac{q^{m}-1}{q-1}}\right\rangle$ be the subgroup of order $q-1$ of $B_{3}, B_{3}^{\prime}=\left\{g C_{3} \mid g \in B_{3}\right\}$ the left coset of $C_{3}$ in $B_{3}$. Therefore, $\left|B_{3}^{\prime}\right|=\frac{q^{m}-1}{q-1}$. Furthermore, the cyclic sets $A_{3}$ and $B_{3}^{\prime}$ are chosen so that $A_{3} \cap B_{3}^{\prime}=\{1\}$. Hence, from Lemma 5, it follows that $A_{3}$ and $B_{3}^{\prime}$ have MLSs.

Now, let $S_{3}=\left\{W_{i} \mid 0 \leq i \leq q^{m}\right\}$ be the classical spread as described in Remark 1 $W_{i}$ be $m$-dimensional totally isotropic subspaces of $V$ for $0 \leq i \leq$ $q^{m}$. Therefore, the partial spread $S_{3}$ partitions the set of all isotropic points of $P(V)$.

We observe that the group $A_{3}$ is sharply transitive on $S_{3}$ with respect to $W_{3}$. Also, $B_{3}^{\prime}$ is sharply transitive on $P\left(W_{3}\right)$ with respect to $\left\langle e_{1}\right\rangle$, where $e_{1} \in W_{3}$.

In case, when $G^{*}=S O_{2 m+1}(q)$, let $a_{3}^{*}=x_{3}^{* q^{m}-1} \in S O_{2 m+1}(q)$ be the matrix corresponding to the linear transformation $T_{\alpha^{q^{m}}-1}$, where $x_{3}^{*}=\left(\begin{array}{cc}x^{*} & 0 \\ 0 & 1\end{array}\right)$, $x^{*} \in S O_{2 m}^{ \pm}(q)$. Let $W_{3}=\left\{e_{1}, e_{2}, \cdots, e_{m}\right\}$ be an $m$-dimensional totally isotropic subspace of $V$ and $D_{3}^{*} \in O_{m}(q) \leq G L\left(W_{3}\right)$. Then, $b_{3}^{*} \in S O_{2 m+1}(q)$ is defined as follows: 23 .

$$
b_{3}=\left(\begin{array}{ccc}
D_{3}^{*} & 0 & 0 \\
0 & D_{3}^{* t} & 0 \\
0 & 0 & 1
\end{array}\right)
$$

From [1, 23, we have that $a_{3}^{*}, b_{3}^{*} \in G$. Let $A_{3}^{*}=\left\langle a_{3}^{*}\right\rangle, B_{3}^{*}=\left\langle b_{3}^{*}\right\rangle$ be cyclic subgroups of $G$. Then, $\left|A_{3}^{*}\right|=q^{m}+1$ and $\left|B_{3}^{*}\right|=q^{m}-1$. 
Let $C_{3}^{*}=\left\langle b_{3}^{* \frac{q^{m}-1}{q-1}}\right\rangle$ be the subgroup of order $q-1$ of $B_{3}^{*}$ and $B_{3}^{*}=\left\{g C_{3}^{*} \mid g \in\right.$ $\left.B_{3}^{*}\right\}$ be the left coset of $C_{3}^{*}$ in $B_{3}^{*}$. Thus, $\left|B_{3}^{\prime *}\right|=\frac{q^{m}-1}{q-1}$. Furthermore, the cyclic sets $A_{3}^{*}$ and $B_{3}^{\prime *}$ are chosen so that $A_{3}^{*} \cap B_{3}^{\prime *}=\{1\}$. Then from Lemma [5, it follows that $A_{3}^{*}$ and $B_{3}^{\prime *}$ have MLSs.

Now, let $S_{3}=\left\{W_{i} \mid 0 \leq i \leq q^{m}\right\}$ be the classical spread as described in Remark 1. $W_{i}$ be $m$-dimensional totally isotropic subspaces of $V$ for $0 \leq i \leq$ $q^{m}$. Then, the partial spread $S_{3}$ partitions the set of all isotropic points of $P(V)$.

Moreover, we observe that the group $A_{3}^{*}$ is sharply transitive on $S_{3}$ with respect to $W_{3}$. Also, $B_{3}^{\prime *}$ is sharply transitive on $P\left(W_{3}\right)$ with respect to $\left\langle e_{1}\right\rangle$, where $e_{1} \in W_{3}$. Hence, we have the following lemma.

Lemma 9 Let $A_{3}, B_{3}^{\prime} \subseteq O_{2 m+1}(q)$ (resp. $A_{3}^{*}, B_{3}^{\prime *} \subseteq S O_{2 m+1}(q)$ ), $S_{3}$ be the partial spread, $W_{3}$ be the subspace of $V$, and $w=\left\langle e_{1}\right\rangle$. Then,

(i) $A_{3}$ (resp. $A_{3}^{*}$ ) is a sharply transitive set on $S_{3}$ with respect to $W_{3}$.

(ii) $B_{3}^{\prime}$ (resp. $\left.B_{3}^{\prime *}\right)$ is a sharply transitive set on $P\left(W_{3}\right)$ with respect to $w_{3}$.

Theorem 7 Let $q$ be a power of odd primes. Then, the orthogonal group $\mathrm{O}_{2 m+1}(q)$ has an $M L S$.

Proof Let $G=O_{2 m+1}(q), A=A_{3}, B=B_{3}^{\prime}, w=\left\langle e_{1}\right\rangle$. When $m=0, O_{1}(q) \cong$ $C_{2}$. Lemma 4 implies that $O_{1}(q)$ has an MLS. When $m \geq 1$, from Lemma 5 and Lemma 9. $\left[A_{3}, B_{3}^{\prime}, G_{w}\right]$ is an LS for $G$. Hence, the stabilizer $G_{w}$ is a semidirect product of a $p$-group of order $q^{2 m-1}$ and $G L_{1}(q) \times O_{2 m-1}(q)$. Now from Lemma 4. $p$-groups and $G L_{1}(q)$ have MLSs. Furthermore, by the induction hypothesis, we assume that $O_{2 m-1}(q)$ has an MLS, therefore, $O_{2 m+1}(q)$ also has an MLS. Thus, $G_{w}$ has an MLS. Also, from Lemma 5, the cyclic sets $A_{3}$ and $B_{3}^{\prime}$ have MLSs. Therefore, using Lemma 6, $G$ has an MLS.

Theorem 8 Let $q$ be a power of odd primes. Then, $S O_{2 m+1}(q)$ has an $M L S$.

Proof Let $G^{*}=S O_{2 m+1}(q), A=A_{3}^{*}, B=B_{3}^{* *}, w=\left\langle e_{1}\right\rangle$. In case, when $m=0, S O_{1}(q) \cong C_{2}$. Lemma 4 implies that $S O_{1}(q)$ has an MLS. In case, when $m \geq 1$, from Lemma 5 and Lemma $\left.9 . A_{3}^{*}, B_{3}^{\prime *}, G_{w}\right]$ is an LS for $G$. Then the stabilizer $G_{w}^{*}$ is a semi-direct product of a $p$-group of order $q^{2 m-1}$ and $G L_{1}(q) \times S O_{2 m-1}(q)$. Now from Lemma $4 p$-groups and $G L_{1}(q)$ have MLSs. Furthermore, by the induction hypothesis, we assume that $S O_{2 m-1}(q)$ has an MLS, therefore, $G_{w}^{*}$ has an MLS. Also, from Lemma 5, the cyclic sets $A_{3}^{*}$ and $B_{3}^{\prime *}$ have MLSs. Therefore, using Lemma 6. $S O_{2 m+1}(q)$ has an MLS.

Theorem 9 Let $q$ be a power of odd primes. Then, $P S O_{2 m+1}(q)$ has an $M L S$.

Proof In case, when $G^{*}=S O_{2 m+1}(q)$ and $G^{*}=P S O_{2 m+1}(q)$, let $A=A_{3}^{*}$, $B=B_{3}^{\prime *}, w=\left\langle e_{1}\right\rangle, L$ be the set of all isotropic points of $P(V)$ as described above. Suppose $\eta_{3}: S O_{2 m+1}(q) \rightarrow P S O_{2 m+1}(q) \cong S O_{2 m+1}(q) / Z\left(S O_{2 m+1}(q)\right)$ is the canonical homomorphism onto $P S O_{2 m+1}(q)$, and let $\bar{A}_{3}^{*}=\eta\left(A_{3}^{*}\right)$, $\bar{B}_{3}^{*}=\eta\left(B_{3}^{*}\right)$ and $\overline{G_{w}}=\eta\left(G_{w}\right)$, then $\left[\bar{A}_{3}^{*}, \bar{B}_{3}^{*}, \overline{G_{w}}\right]$ is the corresponding LS 
for $P_{S O} O_{2 m+1}(q)$ from Lemma 2. Also from Section 3, the stabilizer $\overline{G_{w}}$ is a semi-direct product of a $p$-group of order $q^{2 m-1}$ and $G L_{1}(q) \times P S O_{2 m-1}(q)$. Thus, using the same induction as used in Theorem 2, we get that $P S O_{2 m+1}(q)$ has an MLS.

Theorem 10 Let $q$ be a power of odd primes. Then, $P \Omega_{2 m+1}(q)$ has an $M L S$.

Proof In case, when $n=2 m+1$, from [13, we observe that $\Omega_{2 m-1}(q) \cong$ $S p_{2 m-2}(q)$. Also from 23], we have that $S p_{2 m-2}(q)$ has an MLS. Using the induction, we get that $\Omega_{2 m+1}(q)$ has an MLS. Then, we utilize the canonical homomorphism $\theta_{3}: \Omega_{2 m+1}(q) \rightarrow P \Omega_{2 m+1}(q)$ for proving that $P \Omega_{2 m+1}(q)$ has LS . Consequently, using the same induction as used in Section 5 , we get that $P \Omega_{2 m+1}(q)$ has an MLS. Here, we omit the corresponding proof.

\section{Conclusion}

We utilize partial spreads of totally isotropic subspaces, stabilizers of isotopic 1-subspaces and linear transformations in corresponding vector spaces to construct MLSs for $O_{n}(q), S O_{n}(q), P O_{n}(q), P S O_{n}(q), \Omega_{n}(q)$ and $P \Omega_{n}(q)$ with $q$ as a power of odd primes. Meanwhile, our methods can be used to construct MLSs for other finite simple groups.

\section{Acknowledgements}

This work is partially supported by the National Natural Science Foundation of China (NSFC) (Nos.61103198, 61121061,61370194) and the NSFC A3 Foresight Program (No.61161140320)

\section{References}

1. Babai L., Palfy P.P., Saxl J.: On the number of p regular elements in finite simple groups. LMS J. Comput. Math. 12, 82-119 (2009).

2. De Beule J., Klein A., Metsch K., Storme L.: Partial ovoids and partial spreads of classical finite polar spaces. Serdica Math. J. 34, 689-714 (2008).

3. Bohli J.M., Steinwandt R., González Vasco M.I., Martínez C.:Weak keys in $M S T_{1}$. Des. Codes Cryptogr. 37, 509-524 (2005).

4. Dye R.H.: Maximal subgroups of finite orthogonal groups stabilizing spreads of lines. J. Lond. Math. Soc. 33(2), 279-293 (1986).

5. Garrett P.: Buildings and Classical Groups. Chapman and Hall, London (1997).

6. González Vasco M.I., Steinwandt R.: Obstacles in two public key cryptosystems based on group factorizations. Tatra Mt. Math. Publ. 25, 23-37 (2002).

7. Kantor W.M.: Spreads, translation planes and Kerdock sets. I. SIAM J Algebraic Discret. Methods 3, 151-165 (1982).

8. Lempken W., van Trung T.: On minimal logarithmic signatures of finite groups. Exp. Math. 14, 257-269 (2005).

9. Lempken W., Magliveras S.S., Van Trung T., Wei W.: A public key cryptosystem based on non-abelian finite groups. J. Cryptol. 22, 62-74 (2009). 
10. Magliveras S.S.: A cryptosystem from logarithmic signatures of finite groups. In: Proceedings of the 29th Midwest Symposium on Circuits and Systems, pp. 972-975. Elsevier Publishing Company, Amsterdam (1986).

11. Magliveras S.S.: Secret and public-key cryptosystems from group factorizations. Tatra Mt. Math. Publ. 25, 11-22 (2002).

12. Thas J.A.: Ovoids and spreads of finite classical polar spaces. Geom. Dedicata 10, 135-143 (1981).

13. Wilson R.A.: The finite simple groups. Graduate Texts in Mathematics, vol 251. Springer-Verlag, London (2009).

14. Magliveras S.S., Memon N.D: Algebraic properties of cryptosystem PGM. J. Cryptol. 5, 167-183 (1992).

15. Qu M. and Vanstone S. A., Factorizations of elementary abelian p-groups and their cryptographic significance, J. Cryptology, 7 (1994), 201-212.

16. Magliveras S.S., Stinson D.R., Van Trung T.: New approaches to designing public key cryptosystems using one-way functions and trapdoors in finite groups. J. Cryptol. 15 285-297 (2002)

17. Shor P. W.: Polynomial-time algorithms for prime factorization and discrete logarithms on a quantum computer. SIAM Journal on Computing, 26(5):1484-1509 (1997).

18. González Vasco M.I., Rötteler M., Steinwandt R.: On minimal length factorizations of finite groups. Exp. Math. 12, 10-12 (2003).

19. Cossidente A., De Resmini M.J.: Remarks on singer cyclic groups and their normalizers. Des. Codes Cryptogr. 32, 97-102 (2004).

20. Holmes P.E.: On minimal factorisations of sporadic groups. Exp. Math. 13, 435-440 (2004).

21. Singhi N., Singhi N., Magliveras S.S.: Minimal logarithmic signatures for finite groups of lie type. Des. Codes Cryptogr. 55, 243-260 (2010).

22. Svaba P., van Trung T.: Public key cryptosystem $M S T_{3}$ : cryptanalysis and realization, J. Math. Cryptol. 3, 271-315 (2010)

23. Singhi N., Singhi N.: Minimal logarithmic signatures for classical groups. Dec.Codes Cryptogr. 60, 183-195 (2011).

24. Marquardt T., Svaba P., van Trung T.: Pseudorandom number generators based on random covers for finite groups. Des. Codes Cryptogr. 64,209-220 (2012). 